ISSN electrónico: 2445-1355

DOI: http://dx.doi.org/10.14201/fj2020517583

\title{
ORO NANOMÉTRICO Y VECTORIZADO COMO POTENCIAL ESTRATEGIA HACIA EL TRATAMIENTO DE LA ARTRITIS REUMATOIDE
}

\section{Nanometric and vectorized gold as a potential strategy for the treatment of rbeumatoid arthritis}

Inés ORTEGA-TORRES; Carlos ALONSO-MORENO; José Antonio CASTRO-OSMA

Universidad de Castilla-La Mancha, Departamento de Química Inorgánica, Orgánica y Bioquímica-Centro de Innovación en Química Avanzada (ORFEOCINQA), Facultad de Farmacia, 02006-Albacete, Spain

Correo-e: inesortegatorres.iot@gmail.com

RESUMEN: Las nanopartículas de oro han sido utilizadas desde la antigüedad con fines ornamentales y curativos. En las últimas décadas se ha fomentado su estudio en la comunidad científica por sus propiedades físicas, químicas y ópticas únicas, dependientes de tamaño, constituyendo uno de los mayores avances de los últimos años en nanotecnología aplicada en la salud. En la ciencia farmacéutica, las nanopartículas pueden ser herramientas innovadoras para análisis, diagnóstico y procedimientos terapéuticos. En la actualidad, se estudian para emplearse como sistemas de liberación de fármacos para disminuir dosis y con ello efectos adversos, mejorando la biodistribución del fármaco mediante una liberación controlada para tratar patologías, como la artritis reumatoide. Esta patología, de origen desconocido, es tratada con fármacos antirreumáticos modificadores de la enfermedad, como son metotrexato y tocilizumab, cuya finalidad es hacer más lento el proceso, pudiendo llegar hasta detener la evolución de la enfermedad y prevenir un mayor daño de las zonas afectadas. Sin embargo, estos fármacos presentan elevada toxicidad al emplearse durante largos períodos de tiempo. Como alternativa a la terapia actual, este trabajo pretende estudiar la potencial

Ediciones Universidad de Salamanca / @@ $\quad$ FarmaJournal, vol. 5, núm. 1 (2020), pp. 75-83 
mejora en el tratamiento al encapsular el metotrexato en nanopartículas de oro, así como vectorizar estas con tocilizumab.

Palabras clave: nanotecnología; nanopartícula de oro; artritis reumatoide; metotrexato; tocilizumab.

ABSTRACT: Gold nanoparticles have been used since ancient times for ornamental and healing purposes. In the last decades, its study has been promoted by the scientific community due to its unique physical, chemical and optical properties, dependent on size. Recently, this has constituted one of the biggest advances in applied nanotechnology in health. In pharmaceutical science, nanoparticles can be innovative tools for analysis, diagnosis and therapeutic procedures. Nowadays, nanoparticles are used as drug delivery devices in order to reduce either doses or adverse effects since they improve the biodistribution of drugs by making a modified and adapted release to treat diseases such as rheumatoid arthritis. This pathology of unknown origin is treated with disease-modifying anti-rheumatic drugs, such as methotrexate and tocilizumab, whose purpose is to slow down the process, which can even stop the evolution of the disease and prevent further damage to the disease affected areas. However, these drugs have high toxicity when they are used for long periods of time. As an alternative to current therapy, this work aims to study the potential improvement in treatment by encapsulating methotrexate in gold nanoparticles, as well as vectorizing them with tocilizumab.

Key words: nanotechnology; gold nanoparticles; rheumatoid arthritis; methotrexate; tocilizumab.

\section{INTRODUCCIÓN}

La nanotecnología tiene por objeto el desarrollo de estructuras y dispositivos en el rango de tamaño nanométrico (Singh et al., 2009). Se trata de sistemas coloidales con estructura amorfa o cristalina y con dimensiones alrededor de los $100 \mathrm{~nm}$ (Khan et al., 2017). Existen partículas nanométricas de origen natural orgánicas e inorgánicas producidas por erupciones volcánicas, incendios forestales, procesos microbianos, reacciones fotoquímicas y erosiones. Aun desconociendo su estructura nanométrica, comenzaron a emplearse en antiguas civilizaciones por sus propiedades medicinales, ópticas y decorativas. 
Las primeras nanopartículas con aplicación en medicina se propusieron para utilizarse en vacunas como sistemas de liberación prolongada de antígenos. Desde entonces, las nanopartículas se postularon como herramientas para la liberación controlada de fármacos pretendiendo dirigirlos a dianas terapéuticas, aumentando la selectividad del tratamiento, reduciendo dosis y efectos secundarios (Caballero et al., 2017).

Las nanopartículas de oro como vehículos de liberación de fármacos. Las nanopartículas sintéticas liberadoras de fármacos pueden clasificarse en varias categorías según su morfología, tamaño, propiedades físicas y químicas, e incluso por sus aplicaciones (Khan et al., 2017). La clasificación más utilizada se dirime en función de su naturaleza (Richards et al., 2017), siendo orgánicas las nanopartículas poliméricas y liposómicas, entre otras, e inorgánicas los puntos cuánticos y las nanopartículas metálicas, como son los óxidos de hierro y las nanopartículas de oro (Figura 1).

FiguRa 1. Representación de diferentes tipos de nanopartículas sintéticas utilizadas como sistemas liberadores de fármacos (Richards et al., 2017)

\section{NANOPARTÍCULAS ORGÁNICAS}

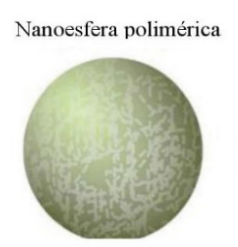

Nanocápsula polimérica
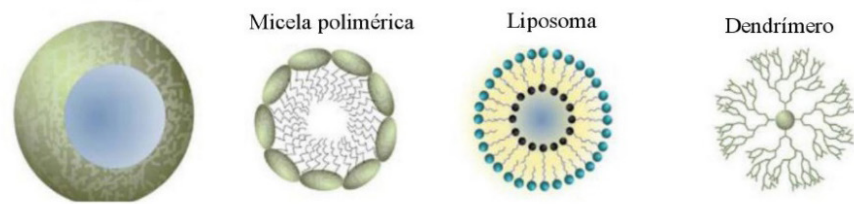

NANOPARTÍCULAS INORGÁNICAS

Nanopartícula de silice mesoporosa
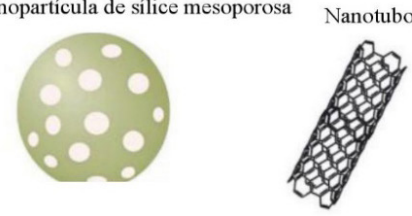

Nanopartícula de óxido de hierro
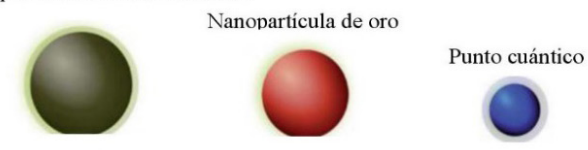

Las nanopartículas metálicas poseen propiedades optoeléctricas y magnéticas únicas (Khan et al., 2017), pudiéndose utilizar para el transporte de fármacos. De entre ellas, destacan las nanopartículas de oro (AuNPs). Estas presentan un núcleo de oro y su tamaño es dependiente de su funcionalización, así como lo son sus propiedades ópticas y químicas (Richards et al., 2017). En cuanto a su aplicación como sistemas liberadores de fármacos, las AuNPs explotan sus propiedades químicas y físicas, mejorando la solubilidad de estos, estabilidad in 
vivo y biodistribución. Mediante el tamaño de AuNPs se controla la velocidad de liberación del fármaco, así las más pequeñas, que tienen una relación volumensuperficie mayor (Mousa et al., 2011), permiten que el fármaco introducido esté cerca de la superficie y se libere de forma más rápida (Singh et al., 2009). En las últimas décadas se han desarrollado diversos enfoques para controlar el tamaño, forma y funcionalidad de la superficie de las AuNPs. Turkevich et al. en 1951 desarrollaron el método más popular mediante la reducción de citrato de $\mathrm{HAuCl}_{4}$ en agua. En 1973 Frens refinó este método para controlar el tamaño de partícula. Brust y Schriffin en 1994 lograron la síntesis de AuNPs de mayor estabilidad, térmicamente estables y de dispersión y tamaño controlado, debido al efecto sinérgico de las interacciones tiol-oro y las atracciones de van der Waals entre ligandos vecinos (Zhao et al., 2013).

Tratamiento de la artritis reumatoide. La artritis reumatoide (AR) es una enfermedad inflamatoria crónica de etiología desconocida y patogénesis multifactorial compleja (Figura 2), caracterizada por daño inflamatorio articular progresivo, inflamación sinovial e hiperplasia irreversible que produce destrucción simétrica y progresiva de las articulaciones y del hueso (Reum Son et al., 2015; Chen et al., 2017). Destacan el factor de necrosis tumoral (TNF- $\alpha$ ), la interleucina 1 (IL-1) y la interleucina 6 (IL-6), que parecen actuar de forma sinérgica en el proceso de inflamación sinovial y destrucción articular, y el factor de crecimiento endotelial vascular (VEGF), importantes para la agravación y la sostenibilidad de la inflamación articular.

Figura 2. Comparación entre una articulación sana y con artritis reumatoide (Mota, 2018)
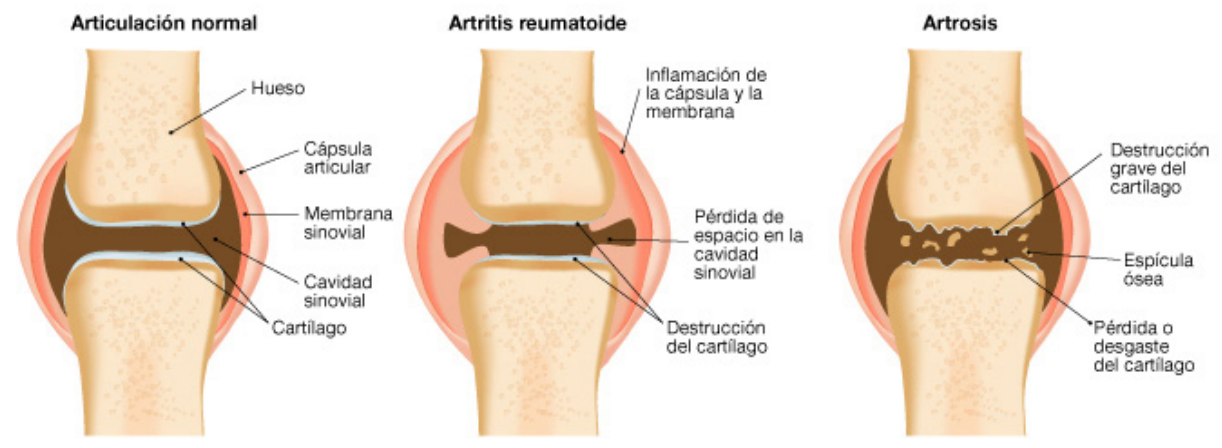

El tratamiento típico de la AR implica el uso de fármacos antirreumáticos modificadores de la enfermedad (FARME), medicamentos de acción lenta que 
evitan o reducen el daño articular (Lee et al., 2013), en la etapa incipiente de la enfermedad. El metotrexato (MTX), fármaco antineoplásico e inmunosupresor antagonista del ácido fólico, que inhibe la síntesis de IL-1, IL-6, TNF-a e interfiere en la síntesis de ADN, es uno de los FARME más frecuentemente utilizados (Reum Son et al., 2015). Este se puede prescribir solo o con otros FARME o agentes biológicos para una terapia combinada. A pesar de su buena eficacia terapéutica, la administración a largo plazo de MTX puede inducir complicaciones sistémicas graves, como infección, hepatitis y supresión de la médula ósea (Lee et al., 2013).

Los avances en la comprensión de la patogénesis revelan objetivos biológicos prometedores (Chen et al., 2017). Por ejemplo, tocilizumab (TCZ), anticuerpo monoclonal humanizado contra el receptor de IL-6. IL-6 es una citoquina pleiotrópica que se sobreexpresa en tejidos sinoviales de pacientes con AR con concentraciones elevadas en suero y líquido sinovial, afecta la función de neutrófilos, células $\mathrm{T}$, células $\mathrm{B}$, monocitos y osteoclastos, que están altamente activados en pacientes con AR (Lee et al., 2014).

\section{2. Овјетіvo}

El objetivo es la encapsulación del fármaco referencia, MTX, sobre AuNPs y su posterior vectorización mediante la conjugación con el TCZ, como alternativa al tratamiento de la AR.

\section{Materiales y mÉtodos}

Productos de partida. Los productos previos para la síntesis de AuNP, como son $\mathrm{HAuCl}_{4}$, citrato trisódico, cisteamina y MTX, N-hidroxisuccinimida (NHS), 1-etil-3-(-3-dimetilaminopropil) carbodiimida (EDC) y células RAW 264.7, se encuentran disponibles comercialmente en la casa Sigma-Aldrich. El TCZ se adquiriría de la empresa Selleck Chemicals.

Síntesis de AuNPs. Las AuNPs se sintetizarían mediante la adición de citrato trisódico a una disolución acuosa de $\mathrm{HAuCl}_{4}$ a $100^{\circ} \mathrm{C}$, acorde al método publicado por Turkevich et al. (Herizchi et al., 2016).

Encapsulación MTX. Para la encapsulación del fármaco MTX en las AuNPs se propone el método descrito por Chao-Liang Wu et al. (Chen et al., 2007).

Caracterización de AuNPs. Las AuNPs se caracterizarían mediante medidas de dispersión de luz dinámica (DLS), para obtener el tamaño medio y la polidispersidad de las partículas, y mediante microscopía electrónica de barrido (SEM) para estudiar su morfología.

La cuantificación de MTX incorporado a la AuNP se realizaría mediante análisis UV-Vis de los sobrenadantes, acorde al trabajo de Tai et al. (Tran et al., 
2013). La curva de calibrado para el MTX se construiría en base al pico de absorción a $372 \mathrm{~nm}$.

La eficiencia de carga (LE) y la eficiencia de encapsulación (EE) se calcularían empleando las siguientes ecuaciones (Reum Son et al., 2015).

$$
\begin{gathered}
L E \%=\frac{\text { masa encapsulada de } \operatorname{MTX}(\mathrm{mg})}{\text { masa del total }(\text { MTX encapsulada }+ \text { masa de AuNPs })(\mathrm{mg})} \times 100 \\
E E \%=\frac{\text { masa encapsulada de } \operatorname{MTX}(\mathrm{mg})}{\text { masa administrada de } \operatorname{MTX}(\mathrm{mg})} \times 100
\end{gathered}
$$

Las mediciones del potencial Zeta mediante DLS darían información de la estabilidad de nuestras formulaciones. El potencial de superficie de la AuNP no conjugado tiene una carga negativa de aproximadamente $-30 \mathrm{mV}$ (Lee et al., 2014). Se esperaría una disminución en el valor del potencial zeta una vez obtenido el complejo MTX-AuNPs (Chen et al., 2007).

Vectorización TCZ. Las AuNPs cargadas con MTX se harían reaccionar con cisteamina y BSA para conseguir nanopartículas cuya superficie estuviera funcionalizada con un grupo amino, requisito indispensable para su conjugación al anticuerpo.

Siguiendo el trabajo de Malhotra et al. (Sharma et al., 2010), las AuNPs funcionalizadas con cisteamina se dispersarían en tampón de fosfato de sodio (PB) a pH 7,4; a continuación, se adicionaría una disolución de TCZ en una relación 1:3 v/v, seguido de reacción con EDC y NHS. Posteriormente, las MTX-AuNPs-TCZ se separarían por centrifugación y se lavarían con PB.

Caracterización MTX-AuNPs-TCZ. La caracterización del complejo MTXAuNPs-TCZ se realizaría mediante DLS y por SEM para determinar el tamaño y forma geométrica de las partículas, respectivamente (Lee et al., 2014). Se llevaría a cabo el ensayo ELISA indirecto para cuantificar TCZ conjugado.

Estudios in vitro. Una vez formadas y caracterizadas las distintas AuNPs, estas se testarían mediante el ensayo MTT (bromuro de 3- (4,5-dimetiltiazol-2-il) -2,5-difeniltetrazolio) sobre líneas celulares RAW 264.7 para evaluar el efecto antiinflamatorio. La viabilidad expuesta a cada tratamiento se determinaría midiendo la conversión del sustrato enzimático soluble en agua MTT en el producto de formazán insoluble en agua de color púrpura en el citoplasma de células RAW 264.7 viables. La evaluación de viabilidad se realizaría utilizando un lector de microplacas. Cada formulación de AuNPs se testaría por triplicado usando diferentes concentraciones y comparando su actividad con MTX libre a 1, 5, 9, 13 y 17 días (Reum Son et al., 2015).

Estudios in vivo. El estudio in vivo se llevaría a cabo mediante la inyección intraarticular en la rodilla de ratas con AR. Para preparar el modelo animal AR, 
se mezclaría una solución 1:1 de colágeno bovino tipo II, adyuvante completo de Freund y se inyectaría en las colas a ratas Lewis macho de 4 semanas de edad. Los protocolos de este estudio fueron aprobados por el Comité Institucional de Experimentos con Animales (Aprobación n. ${ }^{\circ}$ 2013-0070) de la Facultad de Medicina, Universidad de Ajou (Reum Son et al., 2015).

La concentración de MTX-AuNPs inyectada dependería del \%LE, previamente obtenido. En todo momento, se ajustarían las dosis de AuNPs a la de MTX sin encapsular para llevar a cabo la comparativa. Se incluiría la AuNP sin cargar ni vectorizar como control. El control de cambios en los tarsos, tobillo, articulaciones y rodillas de los animales se realizaría mediante fotografías.

\section{Resultados ESPERAdos y discusión}

En este trabajo se pretende proponer la encapsulación MTX sobre AuNPs, que por sí solas presentan efectos beneficiosos contra la AR, y conjugarlas a TCZ, que podría permitir la vectorización del vehículo para una liberación controlada en el lugar de acción, además de actuar como fármaco reduciendo dosis de MTX, disminuyendo toxicidad.

Las AuNPs, MTX-AuNPs y MTX-AuNPs-TCZ serían formuladas acorde a la propuesta de síntesis recogida en el apartado anterior. Estas AuNPs serían caracterizadas de forma adecuada, mediante DLS y microscopía. Las AuNPs deberían tener un tamaño medio alrededor de los $100 \mathrm{~nm}$ con unas polidispersidades cercanas a 0,1 . Los potenciales $\mathrm{Z}$, deberían aproximarse a $-30 \mathrm{mV}$, valor que debería disminuir en valor absoluto una vez cargadas de MTX. Los estudios ELISA nos permitirían confirmar la existencia de anticuerpos en la superficie de las AuNPs.

Una vez obtenidas las AuNPs que contienen MTX, conjugadas y no conjugadas, se procedería, en una primera fase, a realizar estudios in vitro para obtener información sobre viabilidad celular, pudiéndose presentar los siguientes escenarios:

Escenario 1. El empleo de MTX libre a la concentración en estudio en las células RAW 264.7 es significativamente más eficaz que tratar con MTX-AuNPs; por lo tanto, el tratamiento empleado será el de MTX, rechazando la propuesta sugerida.

Escenario 2. El empleo de MTX-AuNPs en las células RAW 264.7 disminuye la viabilidad celular en unos valores mejorados o equiparables al caso de tratar solo con MTX libre. En este caso, se propone la conjugación de MTX-AuNPs al TCZ que pueda obtener mejores resultados en un menor espacio de tiempo. En el caso de que resultados in vitro con MTX-AuNPs-TCZ sean iguales o significativamente mejores a las MTX-AuNPs, estas serán incluidas para estudios in vivo.

Siguiendo las indicaciones de Materiales y métodos, las formulaciones, junto a MTX libre y AuNPs, serían administradas a las distintas ratas, realizando las correspondientes fotografías en 1, 2, 4 y 6 semanas. Esta propuesta sigue el trabajo 
recogido por Reum Son et al. (Reum Son et al., 2015). Los animales con AR no tratados mostrarían edema y eritema severos durante las 6 semanas a estudio. A las 2 semanas, los animales con AR tratados con MTX mostrarían edema y eritema. Sin embargo, estos a las 6 semanas mostrarían rodillas casi normales (ver en Figura 3 el progreso de la patología sin tratamiento y tratada con MTX).

FIgURA 3. Rodillas artríticas de ratón sin tratamiento y con tratamiento con MTX (Reum Son et al., 2015)

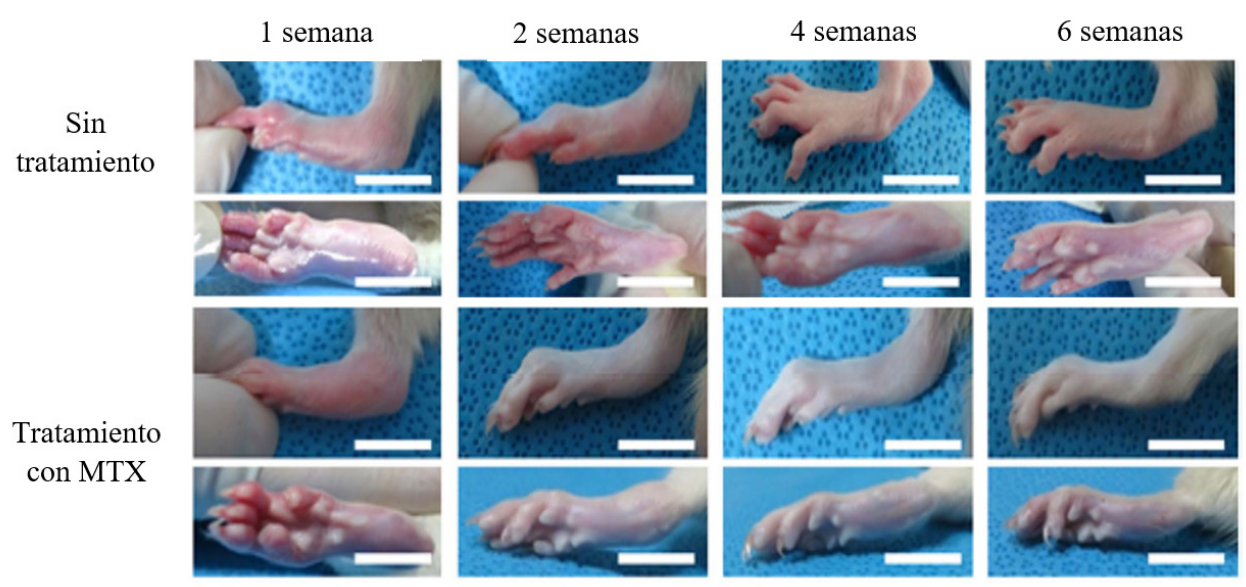

A continuación, se realizarían los ensayos pertinentes mediante la administración in vivo de MTX-AuNPs y MTX-AuNPs-TCZ, realizando un seguimiento por fotografías de los animales en estudio. Podría ocurrir que no hubiera mejora con respecto al MTX sin encapsular, lo que indicaría que nuestra estrategia no sería satisfactoria. En el caso de que las MTX-AuNPs presentaran una mejora en el tratamiento en cuanto a la visualización de edemas y eritemas o en cuanto a su efectividad en el tiempo, indicaría que la vehiculización y liberación controlada sería una propuesta de interés para disminuir la concentración de MTX en el tratamiento de la AR. Por último, se podría esperar que las MTX-AuNPs-TCZ consiguieran el mismo efecto que las MTX-AuNPs en un menor periodo de tiempo, lo que justificaría que la conjugación permite la liberación del fármaco sobre la diana de acción, y que esto podría disminuir los efectos secundarios del tratamiento con MTX sin encapsular. 
I. ORTEGA-TORRES; C. ALONSO-MORENO; J. A.CASTRO-OSMA

ORO NANOMÉTRICO Y VECTORIZADO COMO POTENCIAL ESTRATEGIA HACIA

EL TRATAMIENTO DE LA ARTRITIS REUMATOIDE

\section{BibliografíA}

Caballero AF, Felgueres MJ, Vela E, Gómez D. Estrategias terapéuticas para la artritis reumatoide: hacia las terapias biotecnológicas. Mediagraphic. 2017; 6:69-87.

Chen M, Daddy KA, Xiao Y, Ping Q, Zong L. Advanced nanomedicine for rheumatoid arthritis treatment: focus on active targeting. Expert Opin Drug Deliv. 2017; 14(10):1141-1144.

Chen Y-H, Tsai C-Y, Huang P-Y, Chang M-Y, Cheng P-C, Chou C-H, et al. Methotrexate conjugated to gold nanoparticles inhibits tumor growth in a syngeneic lungtumor model. Mol Pharm. 2007; 4(5):713-722.

Herizchi R, Abbasi E, Milani M, Akbarzadeh A. Current methods for synthesis of gold nanoparticles. Artif Cells, Nanomedicine Biotechnol. 2016; 44(2):596-602.

Khan I, Saeed K, Khan I. Nanoparticles: Properties, applications and toxicities. Arab J Chem. 2017.

Lafarmaciaonline. Cantabria: Manuel Mota Checa; 2008 [actualizado 22 feb. 2018; citado 6 abr. 2018). Disponible en: https://www.lafarmaciaonline.com/es/definicion-sintomasy-como-combatir-la-artrosis-de-rodilla/blog/3/.

Lee H, Lee M-Y, Bhang SH, Kim B-S, Kim YS, Ju JH, et al. Hyaluronate-Gold Nanoparticle/Tocilizumab Complex for the Treatment of Rheumatoid Arthritis. ACS Nano. 2014; 8(5):4790-4798.

Lee SM, Kim HJ, Ha YJ, Park YN, Lee SK, Park YB, et al. Targeted chemo-photothermal treatments of rheumatoid arthritis using gold half-shell multifunctional nanoparticles. ACS Nano. 2013; 7(1):50-57.

Mousa SA, Bharali DJ. Nanotechnology-based detection and targeted therapy in cancer: Nano-bio paradigms and applications. Cancers (Basel). 2011; 3(3):2888-2903.

Reum Son A, Kim DY, Hun Park S, Yong Jang J, Kim K, Ju Kim B, et al. Direct chemotherapeutic dual drug delivery through intra-articular injection for synergistic enhancement of rheumatoid arthritis treatment. Sci Rep. 2015; 5:1-12.

Richards DA, Maruani A, Chudasama V. Chemical Science Antibody fragments as nanoparticle targeting ligands : a step in the right direction. Chem Sci. 2016; 00:1-15.

Sharma A, Matharu Z, Sumana G, Solanki PR, Kim CG, Malhotra BD. Antibody immobilized cysteamine functionalized-gold nanoparticles for aflatoxin detection. Thin Solid Films. 2010; 519(3):1213-1218.

Singh R, Lillard JW. Nanoparticle-based targeted drug delivery. Exp Mol Pathol. 2009; 86(3):215-223.

Tran NTT, Wang TH, Lin CY, Tai Y. (2013). Synthesis of methotrexate-conjugated gold nanoparticles with enhanced cancer therapeutic effect. Biochem. Eng. J. 2013; 78:175-180.

Zhao P, Li N, Astruc D. State of the art in gold nanoparticle synthesis. Coord Chem Rev. 2013; 257(3-4):638-665. 